\title{
Idelalisib induces G1 arrest and apoptosis in chronic myeloid leukemia K562 cells
}

\author{
YALI CHEN $^{1,2}$, QIANXIANG ZHOU ${ }^{1,2}$, LEI ZHANG $^{1,2}$, RAN WANG $^{1}$, \\ MEIHUA JIN $^{1}$, YULING QIU ${ }^{1 *}$ and DEXIN KONG ${ }^{1,2^{*}}$ \\ ${ }^{1}$ Tianjin Key Laboratory on Technologies Enabling Development of Clinical Therapeutics and Diagnostics, \\ School of Pharmacy, and ${ }^{2}$ Research Center of Basic Medical Sciences, \\ Tianjin Medical University, Heping, Tianjin 300070, P.R. China
}

Received May 3, 2016; Accepted June 11, 2016

DOI: 10.3892/or.2016.5176

\begin{abstract}
Increasing resistance of imatinib, a BCR-ABL tyrosine kinase inhibitor, hinders its use in the therapy of chronic myeloid leukemia (CML). The PI3K pathway is known to be closely involved in BCR-ABL transformation and the tumorigenesis of CML, suggesting that PI3K may be a potential target for CML therapy. Idelalisib, a specific inhibitor of PI3K p1108, has been approved for the treatment of chronic lymphocytic leukemia (CLL). However, the antileukemia effect of idelalisib on CML remains unknown. In the present study, the antileukemia activity of idelalisib alone or in combination with imatinib was investigated by use of K562 cells. Idelalisib inhibited K562 proliferation in a dose-dependent manner. G1 arrest was induced, in which upregulation of p27 and p21, as well as downregulation of cyclin D1 and p-pRb, may be involved. Furthermore, idelalisib induced apoptosis in the K562 cells, with increased expression of pro-apoptotic molecules such as Bad and Bax, cleavage of caspase-9, -8 and -3 , and PARP, in contrast to downregulation of anti-apoptotic protein Bcl-2. Combination of idelalisib with imatinib led to a synergistic antiproliferative effect on K562 cells, together with enhanced activity of G1 arrest and apoptosis induction. In conclusion, idelalisib exhibited in vitro antitumor activity on CML K562 cells alone or in combination with imatinib, suggesting potential application in CML therapy.
\end{abstract}

Correspondence to: Professor Dexin Kong or Dr Yuling Qiu, Tianjin Key Laboratory on Technologies Enabling Development of Clinical Therapeutics and Diagnostics, School of Pharmacy, Tianjin Medical University, 22 Qixiangtai Road, Heping, Tianjin 300070, P.R. China

E-mail: kongdexin@tmu.edu.cn

E-mail: qiuyuling@tmu.edu.cn

*Contributed equally

Key words: idelalisib, antileukemia, chronic myeloid leukemia, imatinib, combination

\section{Introduction}

Chronic myeloid leukemia (CML), a clonal myeloproliferative disorder, is characterized by the Philadelphia $(\mathrm{Ph})$ chromosome which originates from the $\mathrm{t}(9 ; 22)(\mathrm{q} 34 ; \mathrm{q} 11)$ reciprocal translocation and leads to the BCR-ABL chimeric oncoprotein (1-3). This BCR-ABL oncoprotein bears constitutive tyrosine kinase activity and therefore promotes uncontrolled growth and proliferation of leukemia cells (4). Development of inhibitors targeting BCR-ABL has been generally successful in the past 15 years, with highly decreased mortality for CML patients. However, resistance to these drugs has been increasingly reported. CML remains an incurable disease $(5,6)$. Therefore, novel drugs with targets other than BCR-ABL are expected.

Idelalisib, also named CAL101, was approved by the US Food and Drug Administration in July, 2014. It is a first-in-class oral PI3K inhibitor that has shown substantial and sustained antileukemia efficacy in patients with relapsed/refractory chronic lymphocytic leukemia (CLL) $(7,8)$. Compared with other chemotherapy regimens, idelalisib showed advantages of long-term efficacy and reduced toxicity (9).

It has been reported that PI3K signaling contributes to BCR-ABL transformation and is essential for in vivo leukemogenesis of CML (10). Furthermore, the PI3K p1108 isoform is preferentially expressed in hematopoietic cells (11), suggesting that idelalisib which targets p1108 shows favorable antitumor efficacy against CML.

Therefore, in the present study, we investigated the antileukemia activities of idelalisib in CML K562 cells.

\section{Materials and methods}

Reagents. Idelalisib and imatinib were purchased from Selleck (London, ON, Canada). 3-(4,5-Dimethyl-2-thiazolyl)2,5-diphenyl-2H-tetrazolium bromide (MTT) reagent was purchased from Amresco (Solon, OH, USA). Antibodies against Akt, phospho-Akt (Ser473), phospho-GSK-3 $\beta$ (Ser9), caspase-3, -8 and -9 , poly(ADP-ribose) polymerase (PARP), $\beta$-actin, and anti-mouse and anti-rabbit HRP-conjugated secondary antibodies were obtained from Cell Signaling Technology (Danvers, MA, USA). Antibodies against phospho-pRb (pS780), cyclin D1 and p27 were obtained from 
BD Biosciences Pharmingen (San Jose, CA, USA). Antibodies against lamin B, p21, Bad, Bcl-2 and Bax were purchased from Santa Cruz Biotechnology (Santa Cruz, CA, USA).

Cell culture. The human CML K562 cell line was purchased from the Cell Resource Center, Peking Union Medical College (Beijing, China). The cells were routinely maintained in RPMI-1640 medium supplemented with $10 \%$ fetal bovine serum, $1 \%$ kanamycin $(100 \mu \mathrm{g} / \mathrm{ml})$, and $1 \%$ glutamine $(0.44 \mu \mathrm{g} / \mathrm{ml})$ at $37^{\circ} \mathrm{C}$ in a humidified atmosphere containing $5 \% \mathrm{CO}_{2}$.

MTT assay. The MTT assay was performed to assess cell viability as previously described $(12,13)$. Briefly, cells $\left(2 \times 10^{4}\right.$ cells $\left./ \mathrm{ml}\right)$ were cultured in a 96-well plate for $48 \mathrm{~h}$ in the presence of $0,1,5,10,20,50,100,150$ and $200 \mu \mathrm{M}$ of idelalisib. After addition of MTT $(5 \mathrm{mg} / \mathrm{ml})$ to each well, the cells were further incubated for $4 \mathrm{~h}$. The produced formazan blue was dissolved with dimethyl sulfoxide (DMSO), and the absorbance was measured at $490 \mathrm{~nm}$ using the microplate reader iMark (Bio-Rad, Hercules, CA, USA).

Soft agar assay. The soft agar assay was carried out as previously described (14) with a small modification. K562 cells were treated with $0,20,50$ and $100 \mu \mathrm{M}$ of idelalisib for $48 \mathrm{~h}$. Then, the treated cells were seeded on solidified agarose in $60-\mathrm{mm}$ dishes $\left(1.2 \times 10^{4}\right.$ cells/dish). After incubation for 10 days at $37^{\circ} \mathrm{C}$, the cells were fixed with $4 \%$ paraformaldehyde and stained with $0.5 \%$ crystal violet for $30 \mathrm{~min}$. Colonies were counted under a microscope. Each assay was performed 3 times.

Flow cytometric analysis of cell cycle distribution. Cell cycle analysis was carried out as previously described (15). The cell suspension $\left(4 \times 10^{5}\right.$ cells $/ 2 \mathrm{ml} /$ well) of $\mathrm{K} 562$ cells was planted in a 6-well plate and exposed to various concentrations of idelalisib for $48 \mathrm{~h}$. The cells were harvested, washed with ice-cold phosphate-buffered saline (PBS), and fixed with 75\% ethanol. After centrifugation, the fixed cells were resuspended in propidium iodide (PI) solution $(25 \mu \mathrm{g} / \mathrm{ml})$, and incubated in the dark for $30 \mathrm{~min}$ at $4^{\circ} \mathrm{C}$ to be available for analysis by BD Accuri C6 flow cytometer (BD Biosciences, San Jose, CA, USA). Data were analyzed using FlowJo 7.6 software.

Flow cytometric analysis of apoptosis with Annexin V-FITC/ PI staining. Analysis of apoptosis was carried out by Annexin V-FITC/PI double staining as previously described (16). K562 cells treated with or without idelalisib in a 6-well plate for $48 \mathrm{~h}$ were collected, washed with ice-cold PBS, and then stained with $2.5 \mu \mathrm{l}$ of Annexin V-FITC and $2.5 \mu \mathrm{l}$ of PI $(5 \mu \mathrm{g} / \mathrm{ml})$ in binding buffer for $15 \mathrm{~min}$ at room temperature in the dark. Flow cytometric analysis was conducted using BD FACSVerse flow cytometer (BD Biosciences).

Synergism assay. Synergism was determined by the isobologram and Fa-CI plot based on Chou and Talalay method $(17,18)$. K562 cells seeded in a 96-well plate were exposed to DMSO (as control), idelalisib, imatinib or their combination at a fixed ratio of $\mathrm{IC}_{50 \text { idelalisib }}$ to $\mathrm{IC}_{50 \text { imatinib }}$ (390:1) for $48 \mathrm{~h}$. Cell growth inhibition was determined using the MTT assay, and the $\mathrm{IC}_{50}$ values were calculated. The combination index (CI) was calculated using CalcuSyn software according to the method of Chou and Talalay: $\mathrm{CI}<1$ is defined as synergism; $\mathrm{CI}=1$ is defined as an additive effect; and CI $>1$ is defined as antagonism. All experiments were carried out in triplicate.

Western blot analysis. Cell lysate preparation and western blot analysis were performed as previously described $(19,20)$. Briefly, total and nuclear proteins were prepared using RIPA lysis buffer (Roche Diagnostics, Basel, Switzerland) and NE-PER Nuclear and Cytoplasmic Extraction kit (Thermo Fisher Scientific, Waltham, MA, USA), respectively. Cell lysates with equal amount of protein were subjected to $10 \%$ SDS-polyacrylamide gel electrophoresis (PAGE), and the separated proteins were transferred onto a polyvinylidene fluoride (PVDF) membrane (Millipore, Billerica, MA, USA). The membrane was blocked in 5\% non-fat dried milk, exposed to the specified primary antibodies overnight at $4^{\circ} \mathrm{C}$, and then to the respective secondary antibodies. The blots were visualized using enhanced chemiluminescence (ECL) reagents and digitalized by scanning.

Statistical analysis. Data are presented as mean \pm standard deviation (SD) from 3 independent experiments. The Student's t-test was carried out for analysis of statistical significance using GraphPad Prism 5 software (GraphPad, San Diego, CA, USA). $\mathrm{p}<0.05$ was regarded as indicative of a statistically significant difference.

\section{Results}

Idelalisib inhibits the proliferation of K562 cells. The inhibitory activity of idelalisib on the proliferation of K562 cells was assessed by MTT and soft agar assays, respectively. First, the MTT assay was utilized. As shown in Fig. 1A, after exposure to idelalisib at concentrations from 1 to $200 \mu \mathrm{M}$ for $48 \mathrm{~h}$, K562 cells showed a dose-dependent decrease in viability, with an $\mathrm{IC}_{50}$ value of $71.4 \mu \mathrm{M}$.

We further examined the antiproliferative activity of idelalisib by use of soft agar assay, which is a suitable method for monitoring anchorage-independent cell growth (21). The cells treated with $0,20,50$ and $100 \mu \mathrm{M}$ of idelalisib for $48 \mathrm{~h}$ were grown in soft agar for 10 days. As shown in Fig. 1B and C, treatment with idelalisib decreased both the number and size of the cell colonies, confirming that idelalisib dose-dependently inhibited K562 cell proliferation.

Idelalisib induces cell cycle arrest in the G1 phase in the K562 cells. To determine whether the suppression of K562 cell proliferation by idelalisib is attributed to cell cycle arrest, cell cycle distribution was examined by flow cytometry after idelalisib treatment for $48 \mathrm{~h}$. As shown in Fig. 2A and B, idelalisib induced accumulation of the cell population in the G1 phase, with $60.8 \%$ for $50 \mu \mathrm{M}$ idelalisib-treated cells vs. $47.4 \%$ for control cells. Then, we investigated the effect of idelalisib on cell cycle-related proteins. Fig. $2 \mathrm{C}$ indicates that treatment with idelalisib decreased the expression of cyclin D1 and the phosphorylation of $\mathrm{pRb}$, but increased the expression of p27 and p21. To further investigate the mechanism of idelalisib in G1 cell cycle arrest, we examined the effect on the phosphorylation of Akt and GSK-3 $\beta$, which are known to regulate the cell cycle downstream of the 

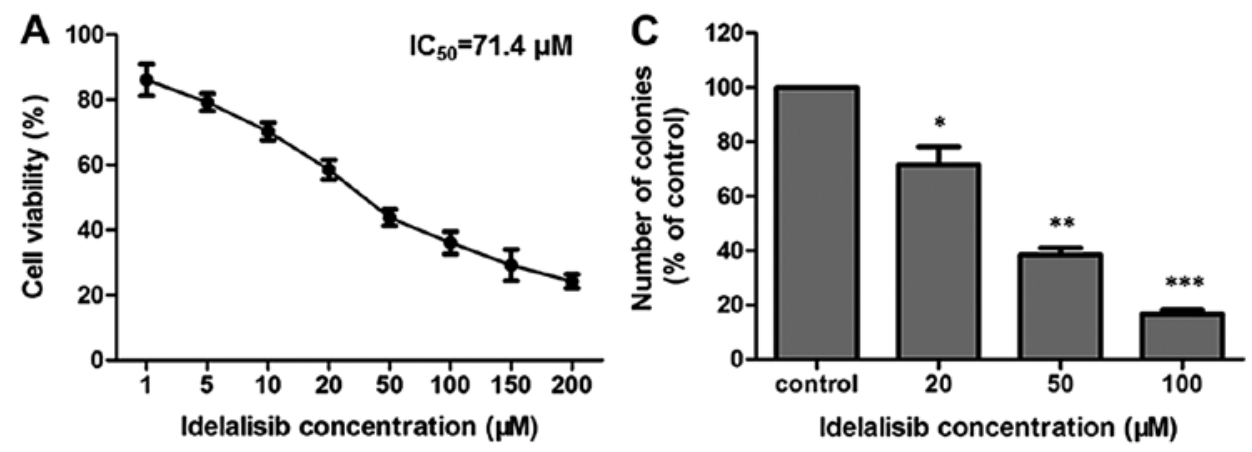

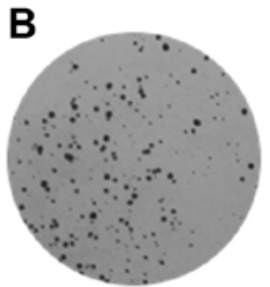

control

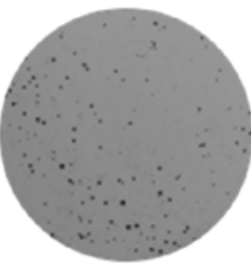

Idelalisib $(20 \mu \mathrm{M})$

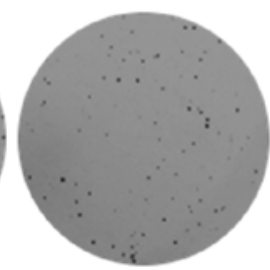

Idelalisib (50 $\mu \mathrm{M})$

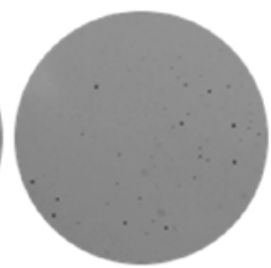

Idelalisib $(\mathbf{1 0 0 \mu \mathrm { M } )}$

Figure 1. Idelalisib inhibits K562 cell proliferation. (A) MTT assay. Cells were exposed to different concentrations $(0,1,5,10,20,50,100,150$ and $200 \mu \mathrm{M})$ of idelalisib for $48 \mathrm{~h}$. Cell viability was measured by determination of the absorbance at $490 \mathrm{~nm}$ after addition of MTT reagent. (B) Soft agar assay. K562 cells treated with different concentrations $(0,20,50$ and $100 \mu \mathrm{M})$ of idelalisib for $48 \mathrm{~h}$, were grown in soft agar for 10 days at $37^{\circ} \mathrm{C}$, fixed with $4 \%$ paraformaldehyde and stained with $0.5 \%$ crystal violet for $30 \mathrm{~min}$. The resulting colonies were counted under a microscope. (C) Bar graph showing the number (percentage of control) of colonies formed by K562 cells with or without idelalisib treatment. Data are mean \pm SD, representative of 3 independent experiments; * $p<0.05$, ${ }^{* *} \mathrm{p}<0.01,{ }^{* * *} \mathrm{p}<0.001$ compared with the control.

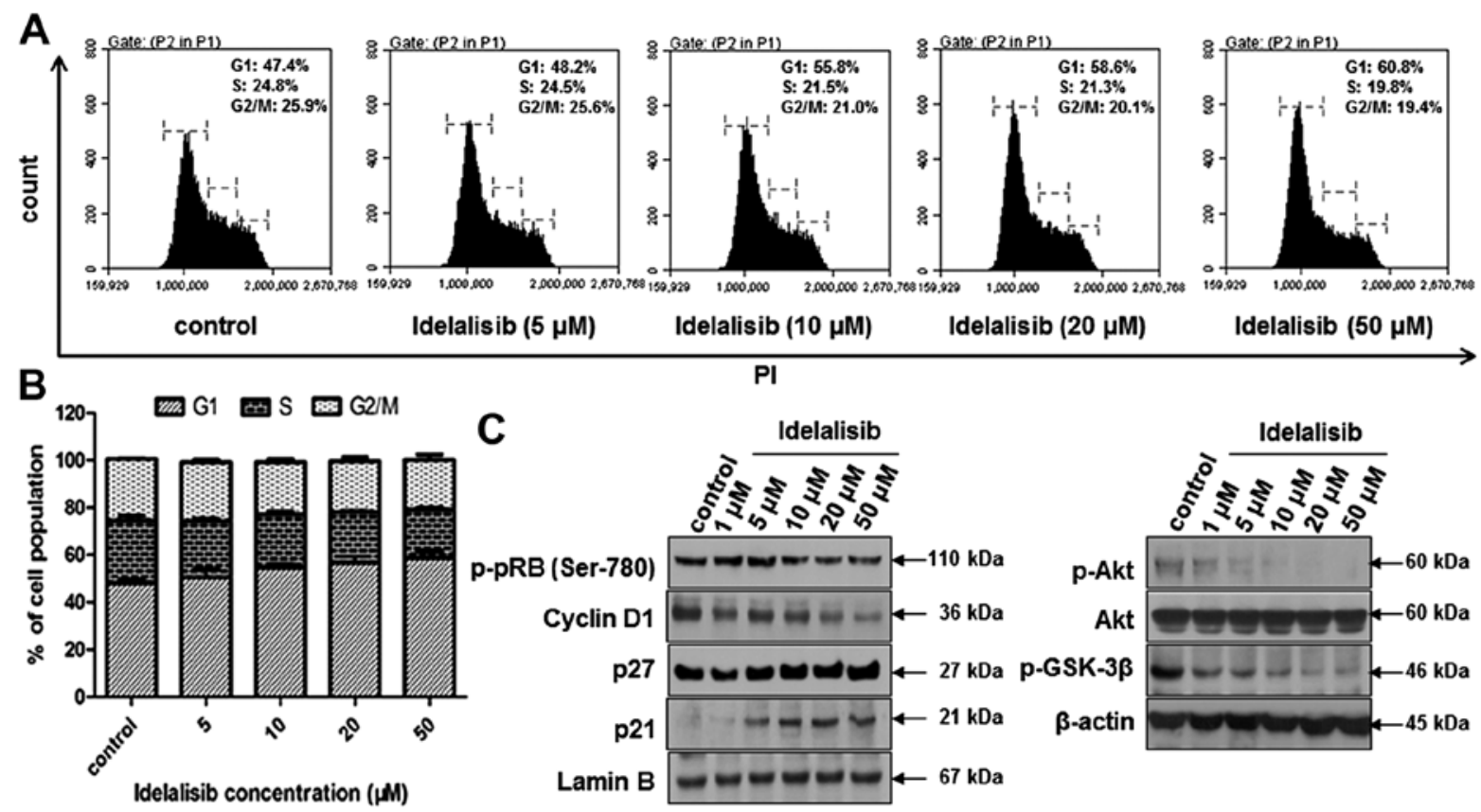

Figure 2. Idelalisib induces K562 cell cycle arrest in the G1 phase. (A) Cell cycle distribution analyzed by flow cytometry. Cells were treated with idelalisib at 0 , 5, 10, 20 and $50 \mu \mathrm{M}$ for $48 \mathrm{~h}$, stained with PI and subjected to flow cytometry. (B) Bar graph showing the percentage of K562 cells in the G1, S and G2/M phase, respectively. Data represent mean \pm SD of 3 independent experiments. (C) Effect of idelalisib on cell cycle-related proteins (left panel) and downstream effectors of the PI3K/Akt pathway (right panel). K562 cells were treated with the indicated concentrations of idelalisib $(0,1,5,10,20$ and $50 \mu \mathrm{M})$ for $48 \mathrm{~h}$. Cell lysates were prepared respectively to be available for analysis of protein levels in the nucleus (p-pRb, cyclin D1, p21 and p27) or whole cell (p-Akt, Akt and p-GSK-3ß) by western blotting.

PI3K/Akt pathway (22). Phosphorylation of Akt and GSK-3 $\beta$ was dose-dependently inhibited by idelalisib, suggesting that the cell cycle arrest effect of idelalisib may be attributed to its blockade of the PI3K/Akt pathway.
Idelalisib induces apoptosis in the K562 cells. Since apoptosis may contribute to a decrease in cell viability, we also examined whether idelalisib induces apoptosis in the K562 cells. Flow cytometric analysis was carried out after Annexin V-FITC/ 
A

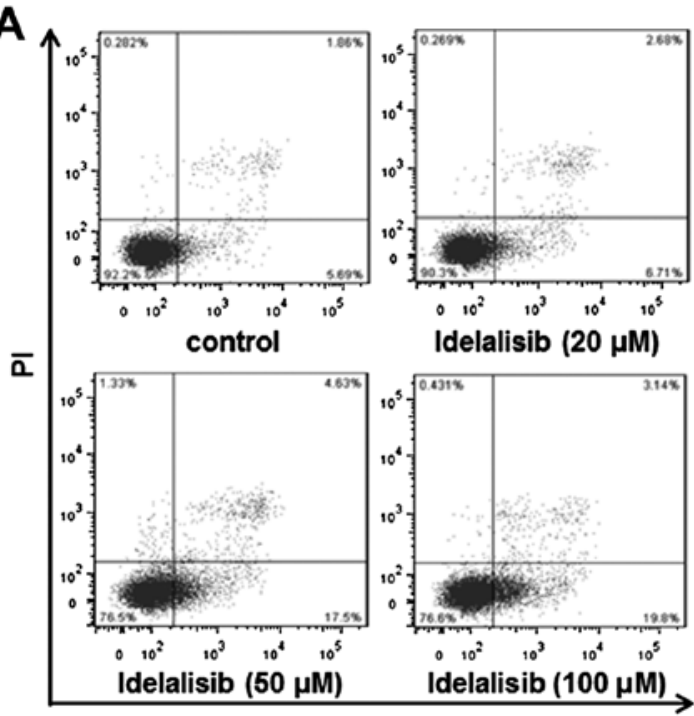

C
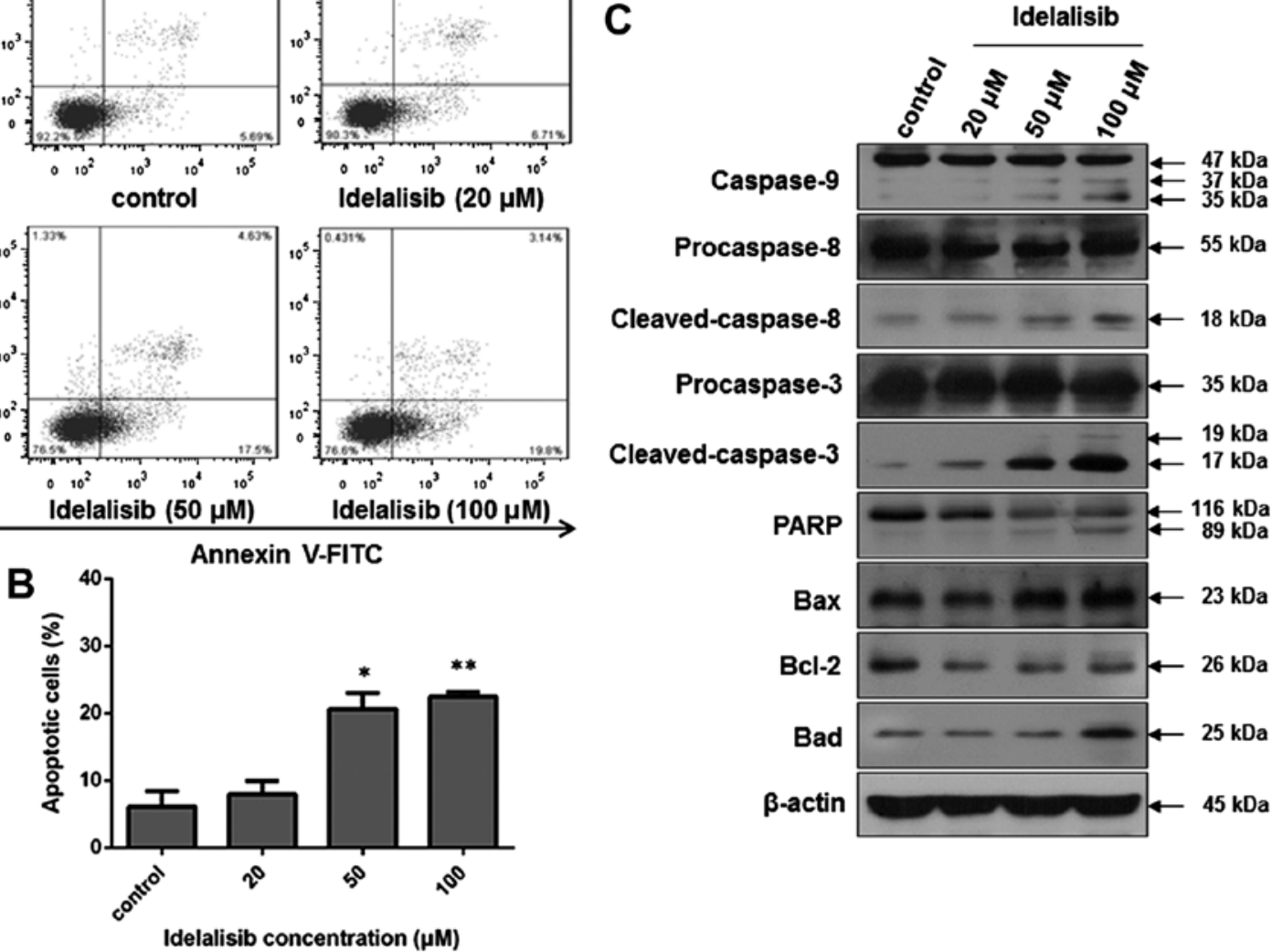

Figure 3. Idelalisib induces apoptosis in the K562 cells. (A) Cell apoptosis analyzed by flow cytometry. Cells were treated with indicated concentrations of idelalisib $(0,20,50$ and $100 \mu \mathrm{M})$ for $48 \mathrm{~h}$, double-stained with Annexin V-FITC/PI and subjected to flow cytometric analysis. (B) Bar graph showing the percentage of apoptotic K562 cells. Data represent mean \pm SD of 3 independent experiments; ${ }^{*} p<0.05,{ }^{* *} p<0.01$, compared with the control. (C) Effect of idelalisib on apoptosis-related proteins. K562 cells were treated with idelalisib $(0,20,50$ and $100 \mu \mathrm{M})$ for $48 \mathrm{~h}$. The levels of cleaved caspase-3,-8 and -9 , and PARP, as well as the expression of Bcl-2, Bax and Bad were examined by western blotting.

PI staining of K562 cells with or without idelalisib treatment. As indicated in Fig. 3A and B, the cell population in the upper- and lower-right quadrants was increased in a dosedependent manner after idelalisib treatment, with $22.94 \%$ for cells treated with $100 \mu \mathrm{M}$ idelalisib vs. $7.55 \%$ for control cells, suggesting that idelalisib induced apoptosis in the K562 cells. Notably, the increased apoptotic cells were mainly in the early-stage (lower-right quadrant; $19.8 \%$ for cells treated with $100 \mu \mathrm{M}$ idelalisib vs. 5.69\% for control cells).

Then, we investigated the effect on apoptosis-related proteins by western blot analysis. As shown in Fig. 3C, idelalisib treatment increased the level of cleaved caspase- $9,-8$ and -3 , and PARP, as well as the expression of Bad and Bax. In contrast, the expression of anti-apoptotic protein Bcl-2 was reduced. These results suggest that the apoptosis induction by idelalisib in K562 cells may be related to the $\mathrm{Bad} / \mathrm{Bcl}-2 / \mathrm{Bax}$ family and the cleavage of caspases and PARP.

Synergistic effect of idelalisib and imatinib in K562 cells. A well-designed drug combination may enhance efficacy while reducing toxicity. To investigate whether idelalisib can enhance the antileukemia activity of the first-line drug imatinib, we carried out a combination study using Chou and Talalay method. K562 cells were treated with idelalisib and imatinib alone or in combination for $48 \mathrm{~h}$, respectively. MTT assay was conducted to determine the inhibitory activities of each drug and the combination. The $\mathrm{IC}_{50}$ of imatinib was calculated to be $0.183 \mu \mathrm{M}$ (Fig. 4A). A synergism study was performed using a series of drug combinations $\left(20,40,60,80\right.$ and $100 \%$ of $\mathrm{IC}_{50}$ value of each drug) with a fixed ratio of $\mathrm{IC}_{50}$ idelalisib to $\mathrm{IC}_{50}$ imatinib (390:1). As shown in Fig. 4B, co-treatment with the two drugs led to an enhanced cell growth inhibition compared to either treatment alone. Analysis of the data by CalcuSyn software indicated a synergistic effect for the combination, since $\mathrm{CI}$ values were $<1$ at all fraction affected (Fa) levels (Fig. 4C). All of the 3 data points $\left(\mathrm{ED}_{50}, \mathrm{ED}_{75}\right.$ and $\left.\mathrm{ED}_{90}\right)$ were far below the additivity line (Fig. 4D), with the respective CI values as $0.62,0.47$ and 0.35 (Table I), suggesting strong synergy of the combination in regards to the growth inhibition of K562 cells.

Then, we further confirmed the combinational effect of idelalisib and imatinib on K562 cells using various assay methods. Soft agar assay showed that co-treatment with idelalisib $(50 \mu \mathrm{M})$ and imatinib $(0.128 \mu \mathrm{M})$ decreased cell proliferation more potently than either drug alone (Fig. 4E and F). Cell cycle distribution analysis indicated an increased G1 arrest (Fig. 5A and B), accompanied by further reduction in the level of $\mathrm{p}-\mathrm{pRb}$ and cyclin $\mathrm{D} 1$, and further enhancement in p21 and p27 expression (Fig. 5C). In addition, 

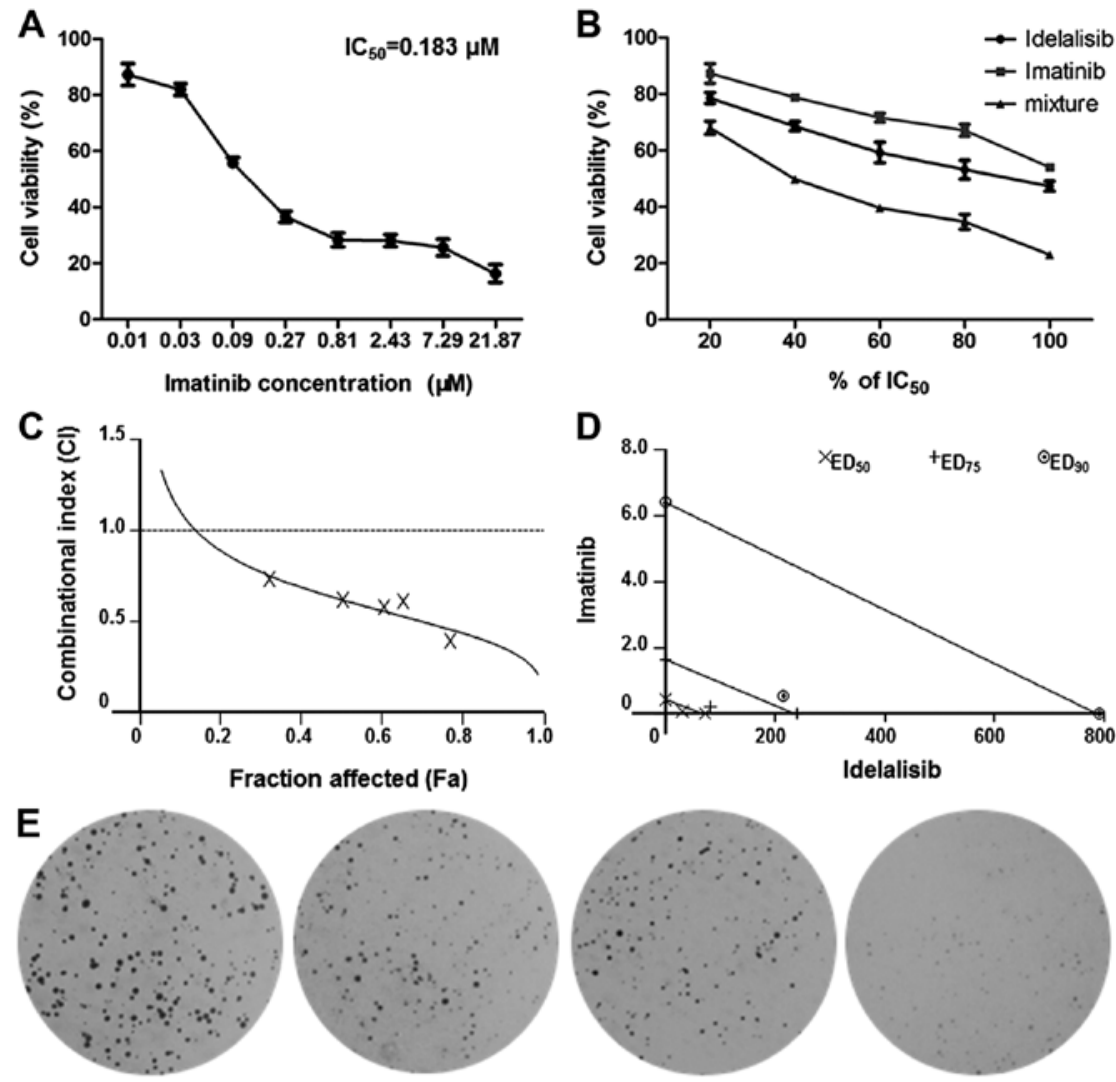

control

Idelalisib

Imatinib

mixture

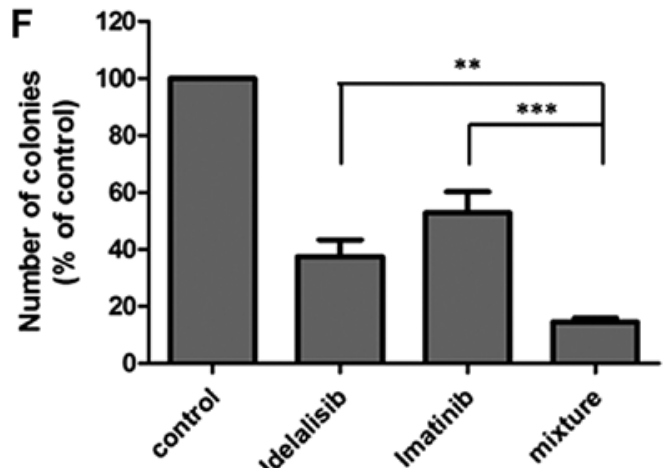

Figure 4. Combination effect of idelalisib and imatinib on K562 cell proliferation. (A) Imatinib inhibited the proliferation of K562 cells. Cells were treated with imatinib $(0,0.01,0.03,0.09,0.27,0.81,2.43,7.29$ and $21.87 \mu \mathrm{M})$. Cell viability was determined by MTT assay. IC $_{50}$ value of imatinib was calculated to be $0.183 \mu \mathrm{M}$. Data are mean $\pm \mathrm{SD}$, representative of 3 independent experiments. (B) Combination with idelalisib enhanced the inhibitory activity of imatinib. K562 cells were exposed to a series of concentrations of idelalisib and imatinib (20,40,60, 80 and $100 \% \mathrm{IC}_{50}$ of each drug), alone or in combination. Cell viability was determined by MTT assay. Data are mean \pm SD, representative of 3 independent experiments. (C) Analysis of the combinational effect using CalcuSyn software. Combinational index (CI) values of drug combinations below the horizontal line (CI=1) represent synergy. Fa, fraction affected. (D) Isobologram of idelalisib and imatinib combination. Data points of growth inhibition at 50\% $\left(\mathrm{ED}_{50}\right), 75 \%\left(\mathrm{ED}_{75}\right)$ and $90 \%\left(\mathrm{ED}_{90}\right)$ are on the left side of the respective lines, indicating a synergistic effect. ( $\mathrm{E}$ and F) Combination of idelalisib and imatinib enhanced the inhibitory activity on K562 proliferation which was determined by soft agar assay. The cells treated with idelalisib $(50 \mu \mathrm{M})$ and imatinib $(0.128 \mu \mathrm{M})$ alone or in combination for $48 \mathrm{~h}$, were grown in soft agar for 10 days at $37^{\circ} \mathrm{C}$. Colonies were counted under a microscope. Data are mean $\pm \mathrm{SD}$, representative of 3 independent experiments; ${ }^{* *} \mathrm{p}<0.01,{ }^{* * * *} \mathrm{p}<0.001$, compared with mixture (combination).

Table I. Combination indices (CI) for idelalisib and imatinib.

CI value $($ mean $\pm \mathrm{SD})$

Cell line

Drug(s)

$\mathrm{IC}_{50}(\mu \mathrm{M})$

r $\quad \mathrm{ED}_{50}$

$\mathrm{ED}_{75}$

$\mathrm{ED}_{90}$

K562

Idelalisib

71.4

0.982

$0.62 \pm 0.09$

$0.47 \pm 0.05$

$0.35 \pm 0.09$

Imatinib

0.183

0.998

Idelalisib + imatinib 


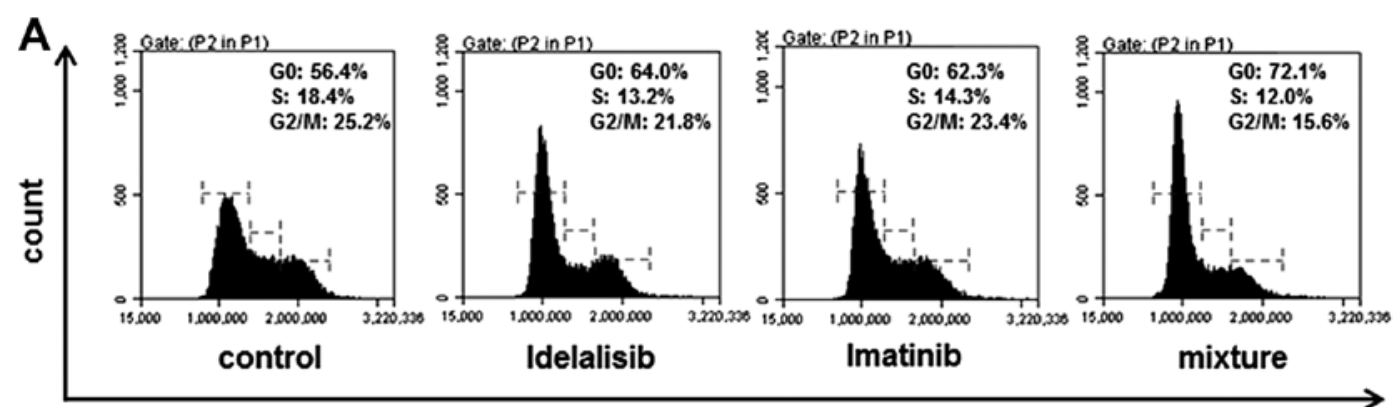

B

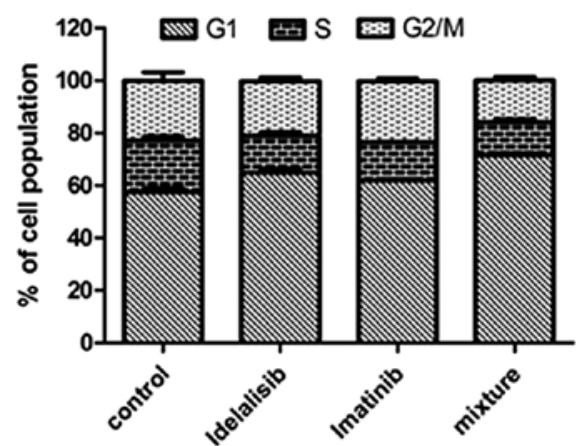

P|

C

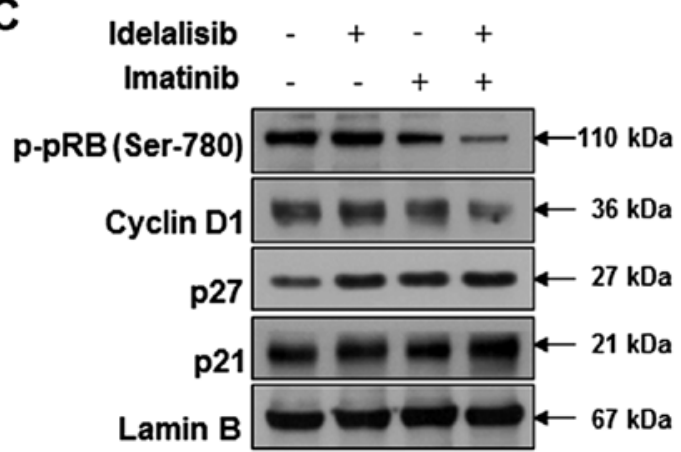

Figure 5. Combination effect of idelalisib and imatinib on K562 cell cycle distribution. (A) Cells treated with idelalisib (50 $\mu \mathrm{M})$, imatinib $(0.128 \mu \mathrm{M})$ or their combination, were analyzed by flow cytometry after PI staining. (B) Bar graph showing the percentage of K562 cells in the G1, S and G2/M phases, respectively. Data represent mean \pm SD of 3 independent experiments. (C) Effect of idelalisib $(50 \mu \mathrm{M})$, imatinib $(0.128 \mu \mathrm{M})$ or their combination, on cell cycle-related proteins. The levels of cyclin D1, p21, p27 and p-pRb in the nucleus of K562 cells after treatment were determined by western blotting.
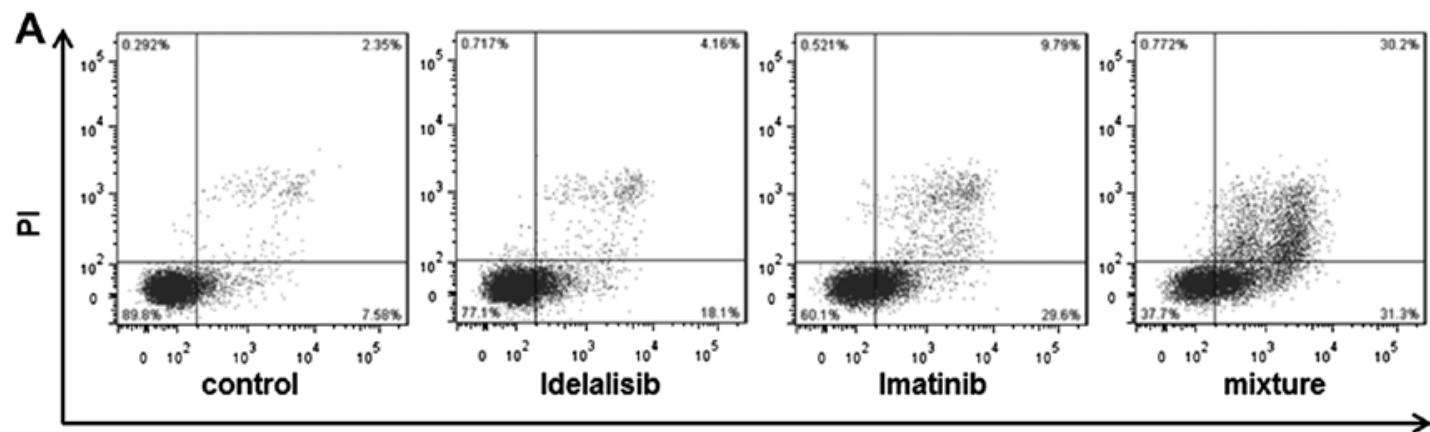

Annexin V-FITC

B

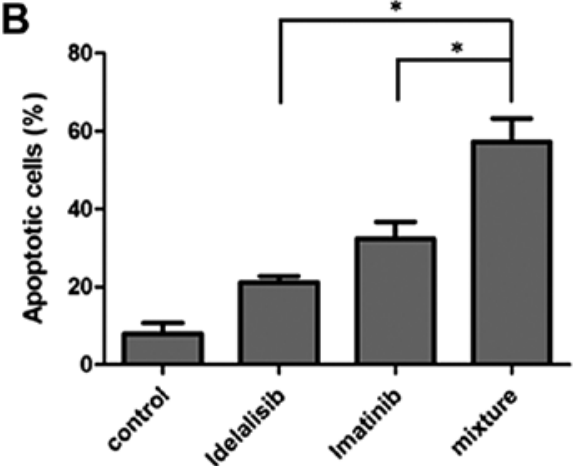

C

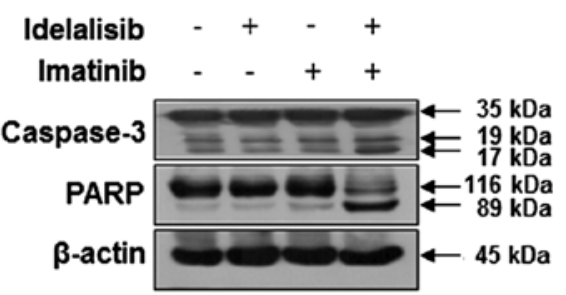

Figure 6. Combination effect of idelalisib and imatinib on K562 cell apoptosis. (A) Cell apoptosis was analyzed by flow cytometry with Annexin V-FITC/ PI staining after treatment with idelalisib $(50 \mu \mathrm{M})$, imatinib $(0.128 \mu \mathrm{M})$ or their combination. (B) Bar graph showing the percentage of apoptotic cells. Data represent mean $\pm \mathrm{SD}$ of 3 independent experiments; ${ }^{*} \mathrm{p}<0.05$, compared with mixture. (C) Effect of idelalisib $(50 \mu \mathrm{M})$, imatinib $(0.128 \mu \mathrm{M})$ or their combination on apoptosis-related proteins. The levels of cleaved caspase-3 and PARP in K562 cells after treatment were examined by western blotting.

combined treatment with idelalisib $(50 \mu \mathrm{M})$ and imatinib $(0.128 \mu \mathrm{M})$ led to increased cell apoptosis than that induced by either drug alone (Fig. 6A and B). Notably, co-treatment with the two drugs induced highly increased cell population 
in both the upper- and lower-right quadrants, suggesting that the combination of idelalisib and imatinib treatment induced apoptosis in both late and early stages. Consistently, the levels of cleaved caspase- 3 and PARP were significantly increased following the combination treatment, as compared with each drug alone (Fig. 6C).

\section{Discussion}

Phosphatidylinositol 3-kinases (PI3Ks), consisting of 3 classes (class I, II and III), are closely involved in cell growth and survival $(23,24)$. Among these 3 classes, class I PI3K is the most studied and is closely related to signaling in hematopoietic cells (25). Class I PI3K is further divided into 2 subtypes as class IA and class IB. Class IA PI3K comprises a regulatory subunit and a catalytic subunit (p110 $\alpha, \mathrm{p} 110 \beta$ or $\mathrm{p} 110 \delta)$, whereas class IB PI3K comprises a p101 regulatory subunit and a $110 \gamma$ catalytic subunit. Catalytic isoform p110 $\delta$ is preferentially expressed in hematopoietic cells (26), suggesting that targeting p110 $\delta$ may be a promising strategy for leukemia therapy. Furthermore, it has been reported that PI3K signaling contributes to BCR-ABL transformation and is essential for leukemogenesis of chronic myeloid leukemia (CML) (10). In addition, sustained activation of the PI3K/Akt signaling pathway may contribute to drug resistance due to enhanced drug efflux by ATP-binding cassette transporters such as P-gp (27,28). Occurrence of resistance has become a big challenge in the chemotherapy of CML in recent years. Therefore, targeting PI3K p1108 may be an alternative approach for CML treatment.

In the present study, the antileukemia activity of idelalisib, a specific inhibitor of PI3K p1108, on CML K562 cells was investigated. Our results demonstrated that idelalisib dose-dependently inhibited K562 cell proliferation, which were supported by MTT and soft agar assays. Both MTT and soft agar assays are well known assays which are used to evaluate cell proliferation in vitro, whereas the latter one can additionally predict tumorigenic ability which is correlated with anchorage-independent growth in vivo (14). G1 cell cycle arrest was induced by idelalisib treatment, accompanied by the decreased expression of cyclin D1 and phosphorylation of $\mathrm{pRb}$ in contrast to the increased expression of cyclin-dependent kinase (CDK)-inhibitors p27 and p21. Meanwhile, idelalisib treatment blocked the phosphorylation of Akt and GSK-3 $\beta$ in a dose-dependent manner. Since Akt is known to promote cell cycle progression by upregulating GSK-3 $\beta$ and cyclin D1 (29), and downregulating CDK inhibitors p27 and p21 (30), the cell cycle arrest effect of idelalisib may be attributed to the blockade of the PI3K/Akt pathway.

Apoptotic cell death is triggered either by the mitochondrial pathway or the death receptor pathway. The former is mainly regulated by the Bcl-2 family which comprises pro-apoptotic proteins such as Bax and Bak, anti-apoptotic proteins such as $\mathrm{Bcl}-2$, and the $\mathrm{BH} 3$-only proteins such as Bad, while the latter is controlled by cell surface death receptors such as Fas (31). In the mitochondrial pathway, Bax and Bak are known to disrupt mitochondrial outer membrane integrity through multimerization and therefore release cytochrome $c$ into the cytosol, which in turn activates caspases including caspase- 9 and -3 , finally leading to apoptosis (32). Bcl-2 exhibits an anti-apoptotic effect by inhibiting formation of the Bax/Bak complex, while Bad promotes apoptosis via neutralizing the inhibitory activity of Bcl-2 on Bax/Bak (32). For the death receptor pathway, Fas induces activation of caspase-8, which cleaves downstream caspases such as caspase-3, promoting apoptosis (31). In the present study, after treatment with idelalisib, expression of Bad and Bax was increased while that of $\mathrm{Bcl}-2$ was reduced; the levels of cleaved caspase-9, -8 and -3 and PARP were enhanced. Akt is known to upregulate Bcl-2 via inhibition of the antagonist Bad in the mitochondrial pathway (33), and to mediate Fas via regulation of FoxO in the cell death receptor pathway (34). Therefore, idelalisib promoted cell apoptosis by activating both the mitochondrial and death receptor pathways, in which targeting PI3K p1108 and the downstream effectors may be involved.

Development of imatinib for CML treatment has met with great success. However, the efficacy has been challenged by the increasing occurrence of acquired resistance, and the original insensitivity of a population of patients. The present study indicates that idelalisib could highly enhance the antileukemia activity of imatinib on K562 cells, suggesting the possibility for the combinational use of the two drugs in the future.

In conclusion, idelalisib, a novel PI $3 \mathrm{~K} \delta$-specific inhibitor, alone or in combination with imatinib, exhibited potential antileukemia activity against CML K562 cells, suggesting the possible future application in the treatment of CML patients.

\section{Acknowledgements}

The present study was supported by grants from the National Natural Science Foundation of China (nos. 81373441, 81202542 and 81402901), the Natural Science Foundation of Tianjin (12JCZDJC25800 and 13JCYBJC24800), the China Postdoctoral Science Foundation Funded Project (2014M551035 and 2014M551037), and the Tianjin Medical University Research Fund (2013ky07).

\section{References}

1. Maru Y: Molecular biology of chronic myeloid leukemia. Cancer Sci 103: 1601-1610, 2012.

2. Holyoake TL and Helgason GV: Do we need more drugs for chronic myeloid leukemia? Immunol Rev 263: 106-123, 2015

3. Rangatia $\mathbf{J}$ and Bonnet D: Transient or long-term silencing of BCR-ABL alone induces cell cycle and proliferation arrest, apoptosis and differentiation. Leukemia 20: 68-76, 2006.

4. Chereda B and Melo JV: Natural course and biology of CML. Ann Hematol 94 (Suppl 2): S107-S121, 2015.

5. Larson RA: Is there a best TKI for chronic phase CML? Blood 126: 2370-2375, 2015.

6. Liu N, Zang S, Liu Y, Wang Y, Li W, Liu Q, Ji M, Ma D and Ji C: FZD7 regulates BMSC-mediated protection of CML cells. Oncotarget 7: 6175-6187, 2016.

7. Brown JR: Idelalisib has CLL on the run! Blood 126: 2656-2657, 2015.

8. Brown JR, Byrd JC, Coutre SE, Benson DM, Flinn IW, Wagner-Johnston ND, Spurgeon SE, Kahl BS, Bello C, Webb HK, et al: Idelalisib, an inhibitor of phosphatidylinositol 3-kinase p1108, for relapsed/refractory chronic lymphocytic leukemia. Blood 123: 3390-3397, 2014.

9. Morabito F, Gentile M, Seymour JF and Polliack A: Ibrutinib, idelalisib and obinutuzumab for the treatment of patients with chronic lymphocytic leukemia: Three new arrows aiming at the target. Leuk Lymphoma 56: 3250-3256, 2015. 
10. Kharas MG, Janes MR, Scarfone VM, Lilly MB, Knight ZA, Shokat KM and Fruman DA: Ablation of PI3K blocks BCR-ABL leukemogenesis in mice, and a dual PI3K/mTOR inhibitor prevents expansion of human $\mathrm{BCR}-\mathrm{ABL}^{+}$leukemia cells. J Clin Invest 118: 3038-3050, 2008.

11. Byrd JC, Woyach JA and Johnson AJ: Translating PI3K-delta inhibitors to the clinic in chronic lymphocytic leukemia: The story of CAL-101 (GS1101). Am Soc Clin Oncol Educ Book 2012: 691-694, 2012. doi: 10.14694/EdBook_AM.2012.32.691.

12. Chen X, Tang SA, Lee E, Qiu Y, Wang R, Duan HQ, Dan S, Jin M and Kong D: IVSE, isolated from Inula japonica, suppresses LPS-induced NO production via NF- $\kappa \mathrm{B}$ and MAPK inactivation in RAW264.7 cells. Life Sci 124: 8-15, 2015.

13. Wang X, Tang SA, Wang R, Qiu Y, Jin M and Kong D: Inhibitory effects of JEUD-38, a new sesquiterpene lactone from Inula japonica thunb, on LPS-induced iNOS expression in RAW264.7 cells. Inflammation 38: 941-948, 2015.

14. Liu Y, Zhang X, Wang J, Yang J and Tan WF: JNK is required for maintaining the tumor-initiating cell-like properties of acquired chemoresistant human cancer cells. Acta Pharmacol Sin 36 1099-1106, 2015.

15. Tang SA, Zhou Q, Guo WZ, Qiu Y, Wang R, Jin M, Zhang W, Li K, Yamori T, Dan S, et al: In vitro antitumor activity of stellettin B, a triterpene from marine sponge Jaspis stellifera, on human glioblastoma cancer SF295 cells. Mar Drugs 12: 4200-4213, 2014.

16. Wang Y, Liu J, Qiu Y, Jin M, Chen X, Fan G, Wang R and Kong D ZSTK474, a specific class I phosphatidylinositol 3-kinase inhibitor, induces G1 arrest and autophagy in human breast cancer MCF-7 cells. Oncotarget 7: 19897-19909, 2016.

17. Chou TC: Drug combination studies and their synergy quantification using the Chou-Talalay method. Cancer Res 70: 440-446, 2010.

18. Radujkovic A, Luft T, Dreger P, Ho AD, Jens Zeller W, Fruehauf S and Topaly J: In vitro testing of drug combinations employing nilotinib and alkylating agents with regard to pretransplant conditioning treatment of advanced-phase chronic myeloid leukemia. Cancer Chemother Pharmacol 74: 427-432, 2014.

19. Kong D, Yamori T, Yamazaki K and Dan S: In vitro multifaceted activities of a specific group of novel phosphatidylinositol 3-kinase inhibitors on hotspot mutant PIK3CA. Invest New Drugs 32: 1134-1143, 2014.

20. Zhao W, Guo W, Zhou Q, Ma SN, Wang R, Qiu Y, Jin M, Duan HQ and Kong D: In vitro antimetastatic effect of phosphatidylinositol 3-kinase inhibitor ZSTK474 on prostate cancer PC3 cells. Int J Mol Sci 14: 13577-13591, 2013.

21. Kusakawa S, Yasuda S, Kuroda T, Kawamata S and Sato Y: Ultra-sensitive detection of tumorigenic cellular impurities in human cell-processed therapeutic products by digital analysis of soft agar colony formation. Sci Rep 5: 17892-17902, 2015.
22. Chang F, Lee JT, Navolanic PM, Steelman LS, Shelton JG, Blalock WL, Franklin RA and McCubrey JA: Involvement of PI3K/Akt pathway in cell cycle progression, apoptosis, and neoplastic transformation: A target for cancer chemotherapy. Leukemia 17: 590-603, 2003.

23. Neri LM, Cani A, Martelli AM, Simioni C, Junghanss C, Tabellini G, Ricci F, Tazzari PL, Pagliaro P, McCubrey JA, et al: Targeting the PI3K/Akt/mTOR signaling pathway in B-precursor acute lymphoblastic leukemia and its therapeutic potential. Leukemia 28: 739-748, 2014.

24. Fruman DA and Rommel C: PI3K and cancer: Lessons, challenges and opportunities. Nat Rev Drug Discov 13: 140-156, 2014.

25. Steelman LS, Pohnert SC, Shelton JG, Franklin RA, Bertrand FE and McCubrey JA: JAK/STAT, Raf/MEK/ERK, PI3K/Akt and $\mathrm{BCR}-\mathrm{ABL}$ in cell cycle progression and leukemogenesis. Leukemia 18: 189-218, 2004.

26. Sujobert P, Bardet V, Cornillet-Lefebvre P, Hayflick JS, Prie N, Verdier F, Vanhaesebroeck B, Muller O, Pesce F, Ifrah $\mathrm{N}$, et al: Essential role for the p110delta isoform in phosphoinositide 3-kinase activation and cell proliferation in acute myeloid leukemia. Blood 106: 1063-1066, 2005.

27. Mayer IA and Arteaga CL: The PI3K/AKT pathway as a target for cancer treatment. Annu Rev Med 67: 11-28, 2016.

28. Sui H, Pan SF, Feng Y, Jin BH, Liu X, Zhou LH, Hou FG, Wang WH, Fu XL, Han ZF, et al: Zuo Jin Wan reverses P-gp-mediated drug-resistance by inhibiting activation of the PI3K/Akt/NF- $\kappa$ B pathway. BMC Complement Altern Med 14: 279-288, 2014

29. Faes S and Dormond O: PI3K and AKT: Unfaithful partners in cancer. Int J Mol Sci 16: 21138-21152, 2015.

30. Warfel NA and Kraft AS: PIM kinase (and Akt) biology and signaling in tumors. Pharmacol Ther 151: 41-49, 2015.

31. Radogna F, Dicato M and Diederich M: Cancer-type-specific crosstalk between autophagy, necroptosis and apoptosis as a pharmacological target. Biochem Pharmacol 94: 1-11, 2015.

32. Green DR and Llambi F: Cell death signaling. Cold Spring Harb Perspect Biol 7: a006080, 2015.

33. Ren C, Ren T, Yang K, Wang S, Bao X, Zhang F and Guo W: Inhibition of SOX2 induces cell apoptosis and G1/S arrest in Ewing's sarcoma through the PI3K/Akt pathway. J Exp Clin Cancer Res 35: 44-57, 2016.

34. Zhang X, Tang N, Hadden TJ and Rishi AK: Akt, FoxO and regulation of apoptosis. Biochim Biophys Acta 1813: 1978-1986, 2011. 